\title{
Band Structure and Broadband Compensation of Absorption by Amplification in Layered Optical Metamaterials
}

\author{
N. N. Rozanov ${ }^{a, b}, *$, S. V. Fedorov ${ }^{a, b}$, R. S. Savel'ev ${ }^{a}$, A. A. Sukhorukov ${ }^{a, c}$, and Yu. S. Kivshar ${ }^{a, c}$ \\ ${ }^{a}$ St. Petersburg State University of Information Technologies, Mechanics and Optics, St. Petersburg, 197101 Russia \\ ${ }^{b}$ Vavilov State Optical Institute, Federal State Unitary Enterprise, St. Petersburg, 199034 Russia \\ ${ }^{c}$ Nonlinear Physics Center, Australian National University, ACT 0200, Canberra, Australia \\ e-mail:nrosanov@yahoo.com \\ Received August 14, 2011
}

\begin{abstract}
The frequency dependence of the gain required to compensate for absorption is determined for a layered structure consisting of alternating absorbing and amplifying layers. It is shown that the fulfillment of the same conditions is required for the existence of a band structure consisting of alternating bands allowed and forbidden for optical radiation propagation in the frequency-wave vector parametric region. Conditions are found under which the gain required for compensation is smaller than thresholds for absolute (parasitic lasing) and convective (waveguide amplification of radiation) instabilities.
\end{abstract}

DOI: $10.1134 / \mathrm{S} 1063776112040140$

\section{INTRODUCTION}

Both classical (electrodynamic, acoustic, etc.) and quantum (electron wavefunction in crystals) waves propagating in media periodic in one or several directions acquire new properties which differ fundamentally from those inherent in homogeneous media. One of the main properties is the appearance of allowed bands and forbidden gaps for the propagation of waves in the frequency-generalized wave vector dependencies [1]. Studies in this direction have been mainly performed for transparent media; however, the consideration of absorption and/or amplification in periodic media is quite important for optics. Examples are distributed feedback lasers [2] and active waveguide periodic structures in which dissipative Bragg solitons can be formed [3]. Of special interest are photonic crystals and layered materials whose efficient operation is restricted by considerable losses, which should be compensated by introducing amplification [4]. Currently, considerable success has been achieved in the development of methods for introducing amplifying layers into metamaterials [5-8], which motivates further investigations of schemes with active metamaterials.

In this paper, we analyze the linear propagation of optical radiation in a system consisting of alternating absorbing and amplifying layers. Linearity of the regime is achieved if the radiation intensity in the medium is lower than the saturation intensity in this medium, and such regimes are required for a number of metamaterial applications, including superresolution [5]. As we will see, the presence of dissipationabsorption and amplification fundamentally changes the type of propagation of waves in such media and, in particular, the concept of allowed and forbidden bands itself. We will show that bands with the zero attenuation coefficient in this scheme are realized when absorption is compensated by amplification. Another feature of the amplifying media is the possibility of appearance of instabilities resulting in the generation or unlimited amplification of spontaneous emission in infinite amplifying media [9]. The compensation problem can be considered solved if amplification in the system remains below the instability development threshold, and in this paper we determine conditions for satisfying this requirement.

\section{WAVES IN A SYSTEM OF AMPLIFYING AND ABSORBING LAYERS}

Consider an isotropic nonmagnetic medium (permeability $\mu=1$ ) with complex linear permittivity $\tilde{\varepsilon}(z)$ and monochromatic electromagnetic radiation at frequency $\omega$. We will mainly analyze the case of $s$-polarization (the electric field strength is $\mathbf{E}=$ $\mathbf{e}_{y} \operatorname{Re}\left\{E(z) \exp \left(i k_{x} n_{0} x-i \omega t\right)\right\}$, where $\mathbf{e}_{y}$ is the unit vector directed along Cartesian coordinate $y, k_{x} n_{0}$ is the $x$ component of the wave vector, and $n_{0}$ is the refractive index of a transparent and homogeneous medium for $z \longrightarrow-\infty)$. The corresponding one-dimensional Helmholtz equation for the complex field amplitude $E(z)$ has the form ( $c$ is the speed of light in vacuum)

$$
\frac{d^{2} E}{d z^{2}}+\left[\frac{\omega^{2}}{c^{2}} \tilde{\varepsilon}(z)-k_{x}^{2} n_{0}^{2}\right] E=0
$$

However, to verify the stability of the $s$-polarization regime, it is also necessary to consider perturbations with $p$-polarization. For the latter in the linear regime, 
it is more convenient to perform transformations with the magnetic field strength $\mathbf{H}=\mathbf{e}_{y} \operatorname{Re}\left\{H(z) \exp \left(i k_{x} n_{0} x-\right.\right.$ $i \omega t)\}$. In this case, the analog of Eq. (1) contains an additional term $[10,11]$ :

$$
\frac{d^{2} H}{d z^{2}}-\frac{d \ln \tilde{\varepsilon}}{d z} \frac{d H}{d z}+\left[\frac{\omega^{2}}{c^{2}} \tilde{\varepsilon}(z)-k_{x}^{2} n_{0}^{2}\right] H=0 .
$$

The medium is a system of plane-parallel layers unbounded in the transverse direction and separated by coordinates $z_{0}, z_{1}, z_{2}, \ldots, z_{N}$. The external layers $(z<$ $z_{0}$ and $\left.z>z_{N}\right)$ are semi-infinite, while the internal layers have the thickness $d_{j}=z_{j}-z_{j-1}, i=1,2, \ldots, N$, $z_{-1}=-\infty$, and $z_{N+1}=+\infty$. The permittivity is $\tilde{\varepsilon}(z)=$ $\tilde{\varepsilon}_{j}=\varepsilon_{j}+i e_{j}$ if $z_{j-1}<z<z_{j}$, where $\varepsilon_{j}$ and $e_{j}$ are the frequency-dependent real and imaginary parts of the permittivity (for real positive frequencies, $e_{j}>0$ for absorbing layers and $e_{j}<0$ for amplifying layers). The complex refractive index is $\tilde{n}_{j}=\sqrt{\tilde{\varepsilon}_{j}}=n_{j}+i \kappa_{j}$, where the branch of the square root is chosen such that the signs of $\kappa_{j}$ and $e_{j}$ coincide $\left(\varepsilon_{j}=n_{j}^{2}-\kappa_{j}^{2}, e_{j}=2 n_{j} \kappa_{j}, n_{j}>0\right)$. The external layers are assumed transparent, and $e_{0}=0$, $e_{N+1}=0, \varepsilon_{0}>0$, and $\varepsilon_{N+1}>0$ for them. We also assume for simplicity that the refractive indices of external semi-infinite layers coincide, $n_{0}=n_{N+1}>0$.

The medium is homogeneous in each layer, so that solution (1) and (1a) inside the $j$ th layer is a sum of two counterpropagating plane waves $(G=E$ or $H)$ :

$$
\begin{aligned}
& G_{j}(z)=F_{j} \exp \left[i k_{z} \bar{n}_{j}\left(z-z_{j-1}\right)\right] \\
& \quad+B_{j} \exp \left[-i k_{z} \bar{n}_{j}\left(z-z_{j-1}\right)\right] .
\end{aligned}
$$

Here, $k_{z} \bar{n}_{j}=\sqrt{k_{0}^{2} \tilde{n}_{j}^{2}-k_{x}^{2} n_{0}^{2}}, k_{0}=\omega / c$ is the wavenumber in vacuum, and the choice of the root branch for internal layers is not important, generally speaking, because terms in (2) include both variants of the choice (the choice of the sign determines the interpretation of waves corresponding to these terms). The choice of the root branch for external (semi-infinite) layers requires explanation. Indeed, we consider here the propagation of a wave through a layered system for the wave incident on the system from a left external layer $z<z_{0}$. In this case, the incident radiation frequency $\omega$ is real and the propagating waves $\left(k_{x}^{2}<k_{0}^{2}\right)$ are characterized by the angle of incidence $\theta$ (in the left semi-infinite layer). Then, in external layers

$$
\begin{gathered}
G_{0}(z)=F_{0} \exp \left[i k_{z} n_{0}\left(z-z_{0}\right)\right] \\
+B_{0} \exp \left[-i k_{z} n_{0}\left(z-z_{0}\right)\right], \\
G_{N+1}(z)=F_{N+1} \exp \left[i k_{z} \bar{n}_{N+1}\left(z-z_{N}\right)\right], \\
B_{N+1}=0,
\end{gathered}
$$

where $k_{x}=k_{0} \sin \theta, k_{z}=k_{0} \cos \theta, \bar{n}_{j}^{2}=\tilde{n}_{j}^{2}+\left(\tilde{n}_{j}^{2}-\right.$ $\left.n_{0}^{2}\right) \tan ^{2} \theta, F_{0}$ and $B_{0}$ are the amplitudes of the incident and reflected waves, and a wave incident on the system from right is absent. For evanescent waves $\left(k_{x}^{2}>k_{0}^{2}\right)$, fields in external layers have the same form (3), but the root branch for $k_{z}$ is chosen so that $\operatorname{Im} k_{z}>0$. The continuity conditions at the boundaries of layers $E(z)$ and $d E / d z$ for the $s$-polarization, and $H(z)$ and $\varepsilon^{-1} d H / d z$ for $p$-polarization allow us to solve the problem of radiation propagation through a system of absorbing and amplifying media.

Because the system contains amplifying layers having the infinite transverse extension, the propagation regime can be destroyed due to absolute and convective instabilities [12]. Absolute instabilities correspond to the exponential (in the adopted linear approximation) growth of the field amplitude at each spatial point of the system, i.e. to the complex frequency $\omega$ with the positive imaginary part $\operatorname{Im} \omega>0$. In our problem, this means that the lasing threshold is exceeded for longitudinal "laser" modes, for which the transverse wave vector is $k_{x}=0$, while in external layers only waves leaving the system $\left(F_{0}=0, B_{N+1}=0\right)$ exist. At the lasing threshold, $\operatorname{Im} \omega=0$ and the real part of the frequency takes a number of discrete values (different frequencies of longitudinal modes). The amplification threshold is determined by the minimal lasing threshold for different longitudinal modes. Above this threshold even in the absence of incident radiation, lasing appears, which begins from the noise level (spontaneous emission) and is established at the level determined by the nonlinear saturation effect (in this paper, nonlinear regimes, including the established lasing regime, are not considered). Note also that above the threshold, when $\operatorname{Im} \omega>0$, the energy flux in a laser mode in external layers is directed from central layers to periphery, and the amplitude of escaping waves exponentially decreases with increasing $|z|$ (the amplitude of these waves at the threshold is independent of $z$ ).

The convective instability in the system under study corresponds to waveguide modes for which the frequency is real, $\operatorname{Im} \omega=0$ and radiation propagates in the transverse direction with the exponentially increasing amplitude during propagation (amplified spontaneous emission). In this case, the wavenumber is complex. We do not consider here the case of backward waves with opposite directions of the phase and group velocities; therefore, the increase in amplitude for $\operatorname{Re} k_{x}>0$ corresponds to the condition $\operatorname{Im} k_{x}<0$. The instability threshold is achieved when the condition $\operatorname{Im} k_{x}=0$ is fulfilled for at least one value of the real frequency. Above the threshold, the energy flux is directed, as in the case of lasing, from the central layers to the periphery. Generally speaking, convective instability with a continuous frequency spectrum transforms to absolute instability (generation of transverse laser modes, a discrete frequency spectrum) if we take into account the parasitic reflection of waves from layer edges or some transverse inhomogeneities of the system providing a feedback in the transverse direc- 
tion. However, we will not consider such regimes, assuming that parasitic reflection is rather weak. The propagation of the incident radiation through the system is stable only if the threshold is neither achieved for any longitudinal laser mode (the absolute instability is absent) nor for any frequency of amplified spontaneous emission (the convective instability is absent).

\section{TRANSFER MATRIX}

Due to the linearity of the problem, the characteristics of the field in different layers can be interconnected by a transfer matrix. The transfer matrix connecting the electric and magnetic field strengths at layer boundaries is often used [11], but matrices acting on the wave amplitudes $F_{j}$ and $B_{j}$ are more convenient for us because they have a clear physical meaning and can easily be used to formulate the coupled-wave equations (for moving inhomogeneities [13]). Transfer matrices on the $j$ th layer, $\hat{M}_{j}$, and on the total system, $\hat{M}$, are introduced by the relations

$$
\begin{aligned}
& \left(\begin{array}{c}
F_{j} \\
B_{j}
\end{array}\right)=\hat{M}_{j}\left(\begin{array}{c}
F_{j+1} \\
B_{j+1}
\end{array}\right), \\
& \left(\begin{array}{c}
F_{0} \\
B_{0}
\end{array}\right)=\hat{M}\left(\begin{array}{c}
F_{j+1} \\
0
\end{array}\right),
\end{aligned}
$$

$$
\hat{M}=\hat{M}_{0} \times \hat{M}_{1} \times \hat{M}_{2} \times \ldots \times \hat{M}_{N-1} \times \hat{M}_{N} .
$$

The amplitude transmission and reflection coefficients, $\tau$ and $r$, respectively, in the problem of the propagation of a wave through a system are expressed in terms of the transfer matrix elements as

$$
\tau=\frac{F_{N+1}}{F_{0}}=\frac{1}{M_{11}}, \quad r=\frac{B_{0}}{F_{0}}=\frac{M_{21}}{M_{11}} .
$$

The modulus of the transmission and reflection coefficients can exceed unity due to the presence of amplification in the system. Transfer matrices have the form (we assume that $d_{0}=0$ )

$$
\begin{gathered}
\hat{M}_{j}=\left(\begin{array}{cc}
\frac{1}{2}\left(1+\frac{\bar{n}_{j+1}}{\bar{n}_{j}}\right) \exp \left[-i k_{z} \bar{n}_{j} d_{j}\right], & \frac{1}{2}\left(1-\frac{\bar{n}_{j+1}}{\bar{n}_{j}}\right) \exp \left[-i k_{z} \bar{n}_{j} d_{j}\right] \\
\frac{1}{2}\left(1-\frac{\bar{n}_{j+1}}{\bar{n}_{j}}\right) \exp \left[i k_{z} \bar{n}_{j} d_{j}\right], & \frac{1}{2}\left(1+\frac{\bar{n}_{j+1}}{\bar{n}_{j}}\right) \exp \left[i k_{z} \bar{n}_{j} d_{j}\right]
\end{array}\right) \text { (s-polarization), } \\
\hat{M}_{j}=\left(\begin{array}{cc}
\frac{1}{2}\left(1+\frac{\tilde{\varepsilon}_{j} \bar{n}_{j+1}}{\tilde{\varepsilon}_{j+1} \bar{n}_{j}}\right) \exp \left[-i k_{z} \bar{n}_{j} d_{j}\right], \frac{1}{2}\left(1-\frac{\tilde{\varepsilon}_{j} \bar{n}_{j+1}}{\tilde{\varepsilon}_{j+1} \bar{n}_{j}}\right) \exp \left[-i k_{z} \bar{n}_{j} d_{j}\right] \\
\frac{1}{2}\left(1-\frac{\tilde{\varepsilon}_{j} \bar{n}_{j+1}}{\tilde{\varepsilon}_{j+1} \bar{n}_{j}}\right) \exp \left[i k_{z} \bar{n}_{j} d_{j}\right], & \frac{1}{2}\left(1+\frac{\tilde{\varepsilon}_{j} \bar{n}_{j+1}}{\tilde{\varepsilon}_{j+1} \bar{n}_{j}}\right) \exp \left[i k_{z} \bar{n}_{j} d_{j}\right]
\end{array}\right) \text { (p-polarization).}
\end{gathered}
$$

The absolute instability threshold (lasing threshold) corresponds to the conditions $\operatorname{Im} \omega=0, k_{x}=0$, and

$$
M_{11}=0,
$$

whereas for the convective instability, $k_{x} \neq 0$ (the transverse wave vector is complex, whereas the frequency is real even above the threshold) and condition (7) is preserved.

Consider now a system with alternating identical absorbing and amplifying layers, so that the system is obtained by periodic repetition of a pair of layers. We assume that "odd" internal layers (with odd numbers) absorb radiation and have a thickness of $d_{1}$ and the complex permittivity $\tilde{\varepsilon}_{1}=\varepsilon_{1}+i e_{1}, e_{1} \geq 0$, while "even" layers with parameters $d_{2}$ and $\tilde{\varepsilon}_{2}=\varepsilon_{2}+i e_{2}, e_{2} \leq 0$ provide amplification. The repetition period of the structure is $d=d_{1}+d_{2}$. The one-period transfer matrix is
$\hat{M}_{12}=\hat{M}_{1} \times \hat{M}_{2}$. The determinant of this matrix is $\operatorname{Det} \hat{M}_{12}=1$, as follows from (6). The eigenvalues

$$
\lambda_{1,2}=\lambda_{c} \mp i \sqrt{1-\lambda_{c}^{2}}
$$

of the matrix $\hat{M}_{12}$ are found from the quadratic equation $\lambda^{2}-2 \lambda_{c} \lambda+1=0$, in which the quantity $\lambda_{c}$ is expressed in terms of the trace of the matrix $\hat{M}_{12}$ as

$$
\begin{gathered}
\lambda_{c}=\frac{1}{2} \operatorname{Tr} \hat{M}_{12}=\frac{1}{4}\left(\sqrt{\frac{q_{1}}{q_{2}}}+\sqrt{\frac{q_{2}}{q_{1}}}\right)^{2} \\
\times \cos \left[k_{z}\left(d_{1} \bar{n}_{1}+d_{2} \bar{n}_{2}\right)\right] \\
-\frac{1}{4}\left(\sqrt{\frac{q_{1}}{q_{2}}}-\sqrt{\frac{q_{2}}{q_{1}}}\right)^{2} \cos \left[k_{z}\left(d_{1} \bar{n}_{1}-d_{2} \bar{n}_{2}\right)\right],
\end{gathered}
$$

where $q_{j}=\bar{n}_{j} / \tilde{\varepsilon}_{j}$ for the $p$-polarization and $q_{j}=\bar{n}_{j}$ for the $s$-polarization. As polarization changes, the values 

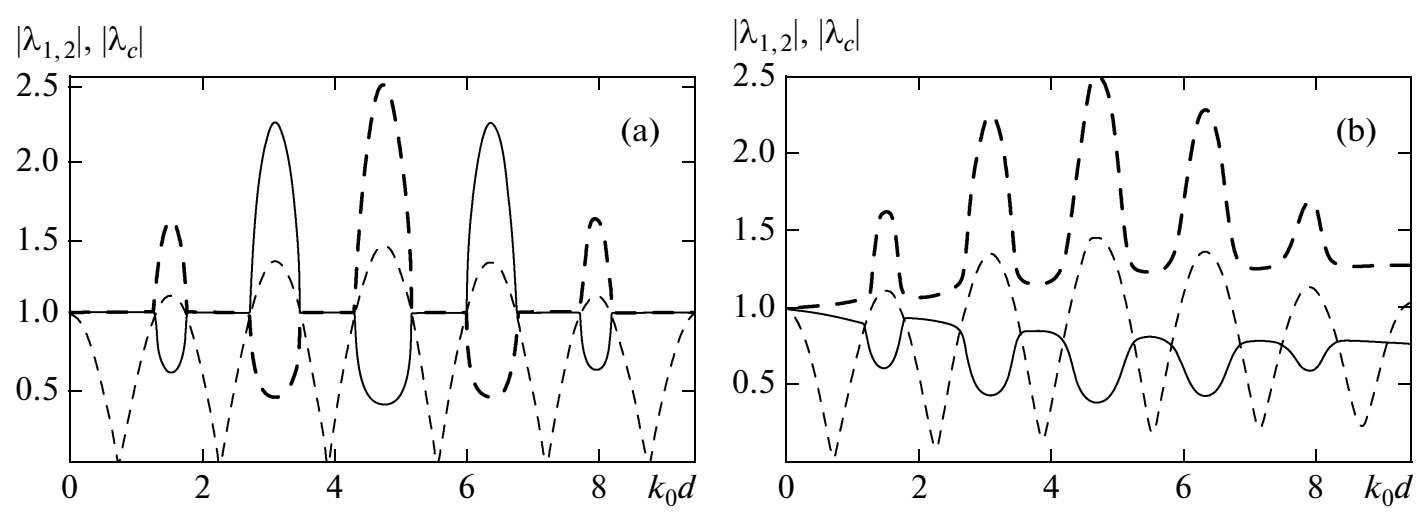

Fig. 1. Frequency dependences of eigenvalue moduli $\left|\lambda_{1,2}\right|$ of transfer matrix $\hat{M}_{12}$ for one period of a structure consisting of two medium layers (solid and heavy dashed curves) and quantities $\left|\lambda_{c}\right|$ (thin dashed curve, $\left|\lambda_{c}\right|>1$ in forbidden bands); $\varepsilon_{1}=1, \varepsilon_{2}=$ $6.25, d_{2} / d_{1}=2$, normal incidence, $e_{2}=0$ (a), \pm 0.2 (b). In the case of ideal compensation for losses, the dependence of type (a) is obtained again.

of $\lambda_{c}$ and diagonal elements $m_{11}$ and $m_{22}$ of the $\hat{M}_{12}$ matrix do not change, while nondiagonal matrix elements change their sign.

The transfer matrix $\hat{M}^{(p)}=\left(\hat{M}_{12}\right)^{p}$ for $p$ identical pairs of layers is calculated by reducing the $\hat{M}_{12}$ matrix to the diagonal form. In particular, its diagonal element is

$$
\begin{aligned}
& M_{11}^{(p)}=\frac{1}{2 \sqrt{1-\lambda_{c}^{2}}}\left(\left(\lambda_{s}+\sqrt{1-\lambda_{c}^{2}}\right)\right. \\
& \left.\times \lambda_{1}^{p}-\left(\lambda_{s}-\sqrt{1-\lambda_{c}^{2}}\right) \lambda_{2}^{p}\right),
\end{aligned}
$$

where

$$
\begin{gathered}
\lambda_{c}=\frac{1}{4}\left(\sqrt{\frac{q_{1}}{q_{2}}}+\sqrt{\frac{q_{2}}{q_{1}}}\right)^{2} \cos \left[k_{z}\left(d_{1} \bar{n}_{1}+d_{2} \bar{n}_{2}\right)\right] \\
-\frac{1}{4}\left(\sqrt{\frac{q_{1}}{q_{2}}}-\sqrt{\frac{q_{2}}{q_{1}}}\right)^{2} \sin \left[k_{z}\left(d_{1} \bar{n}_{1}-d_{2} \bar{n}_{2}\right)\right] .
\end{gathered}
$$

The transfer matrix of the total system with the odd number of layers $N=2 p+1$ has the form $\hat{M}=\hat{M}_{0} \times$ $\hat{M}^{(p)} \times \hat{M}_{N}$. Unbounded photonic crystal or metamaterial can be analyzed by considering the limit for $p \longrightarrow \infty$. Because $\lambda_{1} \lambda_{2}=1$, two cases should be distinguished, analogous to the transparent (without absorption and amplification) periodic structures. In the first case, the moduli of two eigenvalues are different and then one of them necessarily exceeds unity. In this case, according to (9), the matrix element $M_{11}^{(p)} \longrightarrow \infty$ in the limit $p \longrightarrow \infty$, while the transmission coefficient is $\tau_{p}=1 / M_{11}^{(p)} \longrightarrow 0$ (the ideal forbidden band gap). In the second case, the moduli of both eigenvalues are unity, corresponding to the ideal allowed band (the transparency band). The allowed band is realized if two conditions are simultaneously fulfilled: first, the quantity $\lambda_{c}$ should be real and, second, its modulus should not exceed unity:

$$
\operatorname{Im} \lambda_{c}=0, \quad\left|\operatorname{Re} \lambda_{c}\right| \leq 1 .
$$

Violation of at least one of these conditions corresponds to the forbidden band gap.

A periodic structure of transparent layers with the real refractive index contains the allowed and forbidden bands alternating with changing radiation frequency (Fig. 1a). However, in the presence of arbitrarily small absorption, the quantity $\lambda_{c}$ becomes complex, so that, strictly speaking, the allowed and forbidden bands are not defined or the entire spectral range belongs to one forbidden band gap (Fig. 1b). This is natural because radiation completely decays after propagation through an infinite absorbing medium. Note that the replacement of absorption by amplification, when quantities $\bar{n}_{1,2}$ are replaced by their complex conjugate $\left(\bar{n}_{1,2}^{*}\right)$, leads, according to (8), to the complex conjugation of eigenvalues, which, naturally, preserves their moduli. Therefore, the unlimited structure of periodically repeating amplifying layers also proves to be opaque at any radiation frequency (see also [14]).

The latter conclusion may raise doubts because in passing to a homogeneous amplifying medium the transmission is not only nonzero but increases exponentially with increasing thickness of an amplifying layer (in the linear approximation). The discrepancy is explained by the fact that transmission is determined not only by the thickness (the number of periods) of a layered structure but also by the contrast responsible for repeated reflection of waves, which we define in the general case as $\delta=\left(\bar{n}_{2}^{2}-\bar{n}_{1}^{2}\right) / \operatorname{Re} \bar{n}_{1}^{2}$. In a simpler case of the normal incidence, we have $\delta=\left(\tilde{\varepsilon}_{2}-\tilde{\varepsilon}_{1}\right) / \varepsilon_{1}$, i.e. 
this is the contrast of the complex permittivity in periodically alternating layers. The contrast of a homogeneous medium is zero and transmission is

$$
\tau_{p}(0)=\exp \left(i p k_{0} d \sqrt{\varepsilon_{1}+i e_{1}}\right) .
$$

It follows from (9) that a structure can be considered close to homogeneous if $p|\delta| \ll 1$. Note that the bands characterize an unlimited periodic structure $(p \longrightarrow \infty)$, and therefore, it is difficult to say that the band structure is approximately preserved even for small absorption or amplification.

Thus, the ideal allowed bands (transparency bands) are absent in the general case in structures with alternating passive (absorbing) and active (amplifying) layers. However, if passive and active layers exist simultaneously, it is possible to reconstruct the alternating allowed and forbidden bands under conditions when amplification compensates for absorption. Below, we will find these conditions.

Allowed bands or spectral ranges in which the compensation of absorption by amplification is achieved in a multilayered structure are found in the following way. First of all, transparency conditions (11) should be fulfilled for an unlimited structure (in the limit $p \longrightarrow \infty$ ). This can be provided by properly selecting the frequency dependence of the gain in active layers for a specified frequency dependence of the absorption coefficient of passive layers. In addition, for the chosen frequency dependence of the gain, the absolute and convective instability thresholds should not be exceeded. It is natural that the transparency and compensation conditions are determined by many pairs of alternating internal passive and active layers in the structure, whereas the characteristics of a small number of framing layers do not affect the compensation criterion.

In cases important for the problem, when the imaginary part of the permittivity of active and passive layers is noticeably smaller than the real part (small absorption and amplification), the solution of equations is simplified and can be obtained using perturbation theory and the zero-order approximation corresponding to completely transparent media. Thus, the problem with the convective instability in the zeroorder approximation reduces to determining the modes of a planar waveguide with the specified profile of the real permittivity $\varepsilon^{(0)}(z)$, i.e., to determining the field structure in the $E^{(0)}(z)$ mode for $s$-polarization or in the $H^{(0)}(z)$ mode for $p$-polarization (this distribution can be considered real) and the corresponding real eigenvalue $q^{(0)}=\left(k_{x}^{(0)} n_{0}\right)^{2}$ (depending on the parameters, a few local modes can exist) for the specified real frequency $\omega$. In first-order perturbation theory over a small addition $\tilde{\varepsilon}^{(1)}(z)$ to the permittivity, the eigenvalue for the $s$-polarization receives the increment [15]

$$
q^{(1)}=\frac{\omega^{2}}{c^{2}} \frac{\int_{-\infty}^{\infty} \tilde{\varepsilon}^{(1)}(z) E^{(0)^{2}}(z) d z}{\int_{-\infty}^{\infty} E^{(0)^{2}}(z) d z} .
$$

The denominator in the right-hand side of (12) corresponds to the normalization of the waveguide mode field, while the numerator is the integral from amplification and absorption with the field intensity as a weighting function. The instability threshold is achieved for $\operatorname{Im} q^{(1)}=0$. It follows from (12) that the amplification threshold linearly depends on the absorption coefficient in passive layers:

$$
e_{1} \int_{\text {odd }} E^{(0)^{2}}(z) d z+e_{2} \int_{\text {even }} E^{(0)^{2}}(z) d z=0 .
$$

In the presence of absorption in external layers, additional terms appear in (13) which do not change the linear type of the dependence of the amplification threshold on the absorption coefficient. Thus, to find the amplification threshold for perturbations with the $s$-polarization, it is sufficient to solve the waveguide eigenvalue problem in the absence of absorption and amplification and then to calculate integrals in (13) (integration is performed in the first and second terms over passive and active layers, respectively). For perturbations with the $p$-polarization, this approach requires refinements (see Section 4).

\section{NUMERICAL CALCULATIONS}

\subsection{Normal Incidence}

The propagation of $s$-polarized radiation was calculated using the transfer matrix (Section 2). The instability threshold in the form of lasing was found by numerically solving complex equation (7). The instability threshold in the form of waveguide modes in the general case was also determined by numerically solving Eq. (7). In the case of $s$-polarization, the use of perturbation theory described in Section 3 proved efficient. However, in a more complicated case of $p$-polarization, we used, instead of perturbation theory, the direct solution to Eq. (7) by specifying the initial approximation for the roots of the equation corresponding to the real transverse wave vectors for a transparent layered medium.

The main calculation parameters are indicated in the caption to Fig. 1, where the frequency dependences of the moduli of eigenvalues and $\left|\lambda_{c}\right|$ are presented for an infinite periodic system of two transparent (Fig. 1a) and amplifying or absorbing (Fig. 1b) layers. The calculations show that the introduction of 


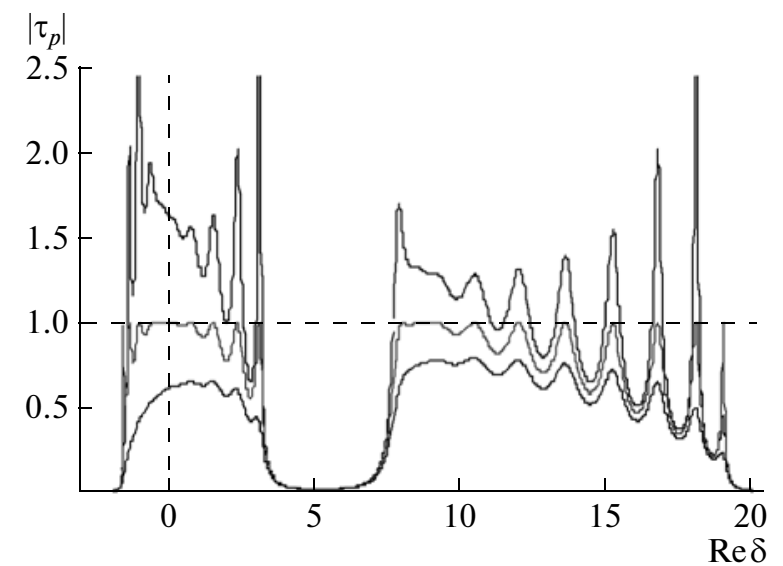

Fig. 2. Dependences of the modulus of the transmission coefficient of the structure containing $p=10$ periods on the contrast of the real part of the permittivity for three values of the imaginary part of the permittivity of even layers: absorption (lower curve, $e_{2}=0.1$ ), transparency (middle curve, $e_{2}=0$ ), and amplification (upper curve, $e_{2}=-0.1$ ); odd layers are transparent $\left(e_{1}=0\right)$. The vertical dashed straight line indicates the zero real part of the contrast.

absorbing or amplifying layers into a structure with a finite number of such pairs weakly changes transmission and reflection in the bands which were forbidden in a structure with transparent media. In this case, the depth of interference oscillations of the transmission and reflection coefficients in the transparency band increases when amplification is introduced and decreases for absorbing layers. However, the maximum of the transmission coefficient considerably changes, exceeding unity for a structure with a finite number of amplifying layers and tending to zero for structures both with absorption and amplification if the number of periods $p$ infinitely increases. This conclusion is confirmed in more detail by the dependences of the transmission coefficient on the contrast of the real part $\tau_{p}(\operatorname{Re} \delta)$ of the permittivity, which are calculated for a fixed radiation frequency, so that $\kappa_{0} d \approx 0.48 \pi$ (Fig. 2). Negative contrasts $\operatorname{Re} \delta<-1$ correspond to the negative real part of the permittivity $\operatorname{Re} \varepsilon_{2}<0$ (metal). Note that the zero real part of the contrast in this case does not mean that the medium is homogeneous because the contrast of the imaginary part of the permittivity is preserved both for the upper and lower curves in Fig. 2. The dependence on the contrast is similar to a certain extent to the frequency dependence, so that the allowed and forbidden bands are observed here already for $p=10$. According to calculations, the presence of the real part of the contrast, i.e., the difference between refractive indices in alternating layers, can considerably increase transmission or reduce reflection compared to a homogeneous medium. However, this occurs only in a selected part of the transparency band degenerating to a point for $p \longrightarrow \infty$. The relative transmission coefficient $\tau_{p}(\delta) / \tau_{p}(0)$ in the main part of

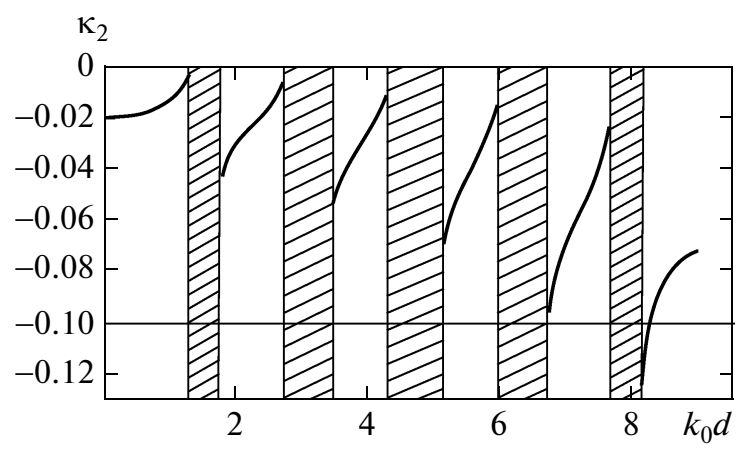

Fig. 3. Frequency dependence of amplification in even layers required for the ideal compensation for absorption in odd layers; $\kappa_{1}=0.1 .\left|\lambda_{1,2}\right|=1$ in allowed bands, like in Fig. 1a. Forbidden band gaps, in which compensation is impossible, are shaded.

the transparency band decreases compared to that for a homogeneous medium layer (for which the contrast is $\delta=0)$.

Now in the presence of absorption in odd layers, we will select amplification in even layers to compensate for absorption for the specified contrast. If we introduce frequency-independent amplification (the amplification band is sufficiently broad), then transmission exists in fact only under conditions when allowed bands are attained for transparent media. At the same time, the transmission coefficient strongly oscillates near the chosen operation frequency. Therefore, we will require the "ideal" compensation, i.e., compensation for any frequency in all transparency bands. The corresponding spectral dependence of $\kappa_{2}$ is shown in Fig. 3. In this case, compensation condition (1) is rigorously fulfilled in spectral ranges close to transparency bands in a system of layers without absorption and amplification. These ranges, which are possible only in the case of ideal compensation, represent the transparency bands of the system under study. The frequency dependence of the eigenvalue moduli $\left|\lambda_{1,2}\right|$ again takes the form shown in Fig. 1a, i.e., alternating allowed bands, in which $\left|\lambda_{1,2}\right|=1$, appear again.

Consider now in detail the frequency dependences of the transmission coefficient of a layered structure for fixed refractive indices $n_{1}=1$ and $n_{2}=2.5$ of alternating layers and absorption coefficient $\kappa_{1}=0.1$ of odd layers, $\tilde{\varepsilon}_{1}=1+0.2 i$. Absorption at a selected frequency in the middle of the transparency band $\left(k_{0} d=\right.$ $0.23 \pi$ ) can be compensated by introducing small amplification in even layers, $\kappa_{2} \approx-0.0174, \tilde{\varepsilon}_{2} \approx 6.25-$ $0.087 i$. The frequency dependences of the transmission, $\left|\tau_{p}\left(k_{z}\right)\right|$, and reflection, $\left|r_{p}\left(k_{z}\right)\right|$, coefficients are shown $n$ Fig. 4 . We can see from the figure that compensation for the number of periods $p=10$ somewhat 

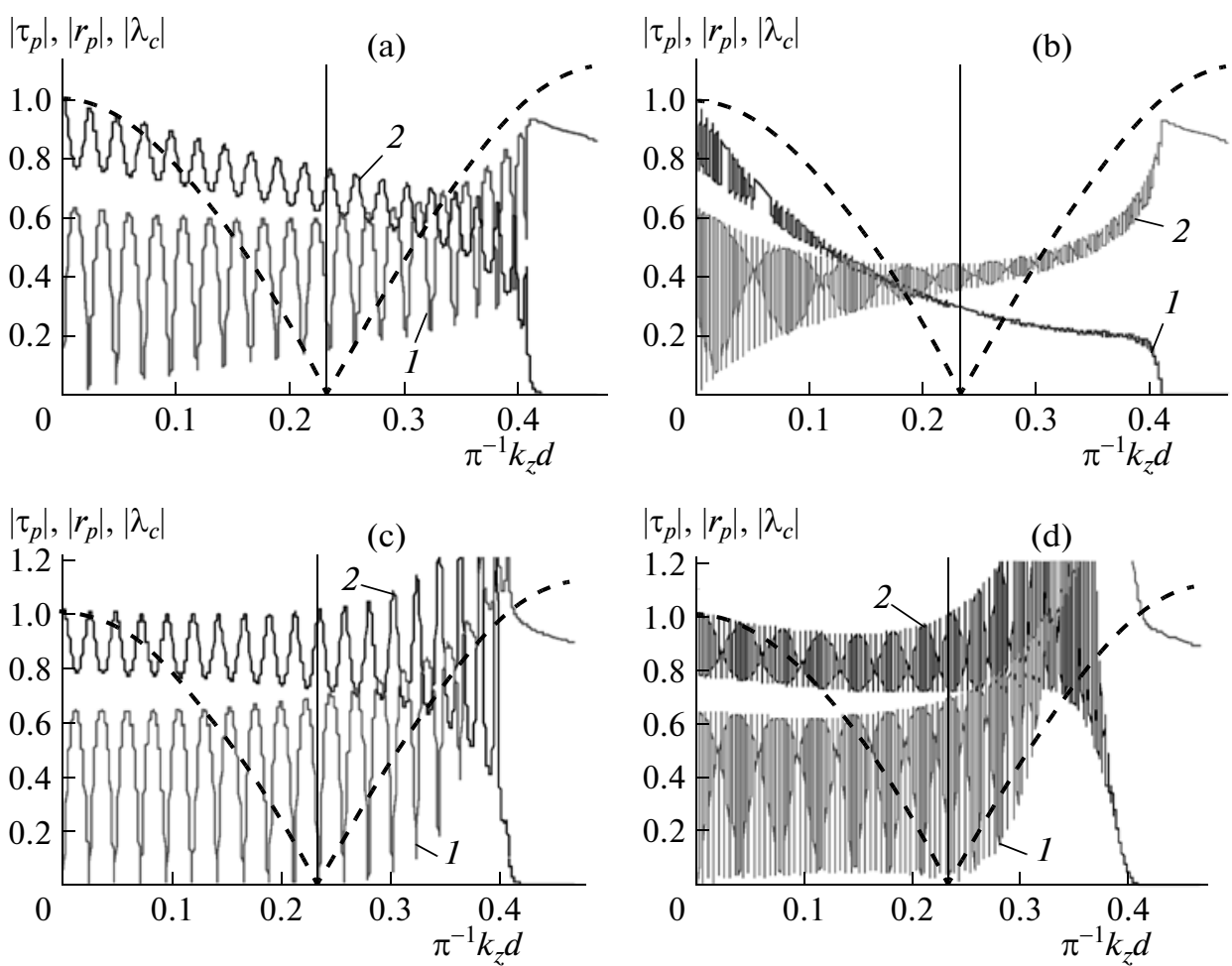

Fig. 4. Dependences of the moduli of the transmission (1) and reflection (2) coefficients on the normalized component of the wave vector $\pi^{-1} k_{z}\left(d_{1}+d_{2}\right)$ for the cases of uncompensated $(\mathrm{a}, \mathrm{b})$ and compensated $(\mathrm{c}, \mathrm{d})$ absorption in the second layer; $p=10$ $(\mathrm{a}, \mathrm{c})$ and $100(\mathrm{~b}, \mathrm{~d})$; the vertical straight line indicates the middle of the transparency band corresponding to the minimum of $\left|\lambda_{c}\right|$ (dashed curve). The case of normal incidence, $k_{z}=k_{0}$.

increases transmission, retaining interference oscillations. For $p=100$, the compensation effect is more pronounced, but the problem of suppressing lasing appears (see Section 4.3 below).

The boundary of the first forbidden band for the specified absorption coefficient $\kappa_{1}=0.1$ in odd layers is determined as the value $k_{0} d=k_{\text {bnd }}$ satisfying the rela-

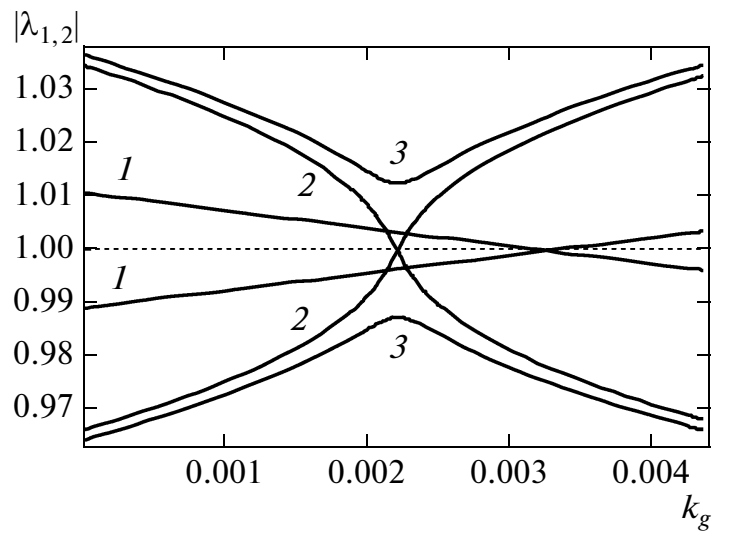

Fig. 5. Dependences of the eigenvalue modulus on amplification in even layers $\kappa_{g}=-\kappa_{2}$ for different frequencies: $k_{0} d=0.99 k_{\mathrm{bnd}}(1), k_{\mathrm{bnd}}(2), 1.0001 k_{\mathrm{bnd}}(3)$. tion $\operatorname{Re}\left[\lambda_{c}\left(k_{0}, \kappa_{1}, \kappa_{2}\right)\right]=1$, but only for the compensation gain value $\kappa_{2}=-\kappa_{g}$, for which the additional condition $\operatorname{Im}\left[\lambda_{c}\left(k_{0}, \kappa_{1},-\kappa_{g}\right)\right]=0$ is valid. For parameters used here, $k_{\mathrm{bnd}}=1.287$. In this case, we can select the gain in even layers in the allowed band $\left(k_{0} d \leq k_{\text {bnd }}\right)$ so that the eigenvalue moduli would be $\left|\lambda_{1,2}\right|=1$, thereby providing compensation. Compensation in the forbidden band $\left(k_{0} d>k_{\mathrm{bnd}}\right)$ is impossible because in this case $\left|\lambda_{1,2}\right| \neq 1$, as follows from Fig. 5 .

The ideal compensation in the form presented here corresponds to the selection of the spectral dependence of the imaginary part of the permittivity (gain) for the specified real part of the permittivity (refractive index). Generally speaking, the causality principle establishes certain relations between the real and imaginary parts of the permittivity (Kramers-Kronig relations) [10], which are somewhat modified in amplifying media. The specific features of the situation under study are as follows. First, absorption and amplification are small; i.e., the imaginary part of the permittivity is small. Second, the gain band within which broadband absorption can be compensated is comparatively narrow. Third, any frequency dependence of the complex permittivity can be approximated with any accuracy within a limited spectral 

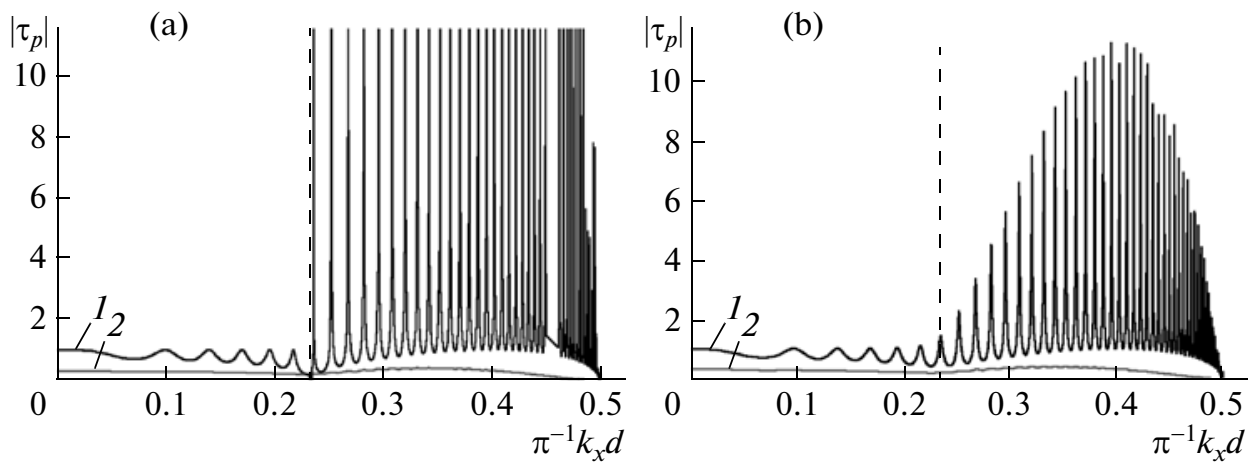

Fig. 6. Dependences of the moduli of the transmission coefficient on the transverse component of the wave vector for a fixed frequency. The region of evanescent waves is located to the right of the vertical dashed straight line. For a system of transparent layers (a, curve 1), poles at selected values of $k_{x}$ correspond to waveguide modes. Curve 2 corresponds to uncompensated absorption, $\kappa_{1}=0.1, \kappa_{2}=0$. Curve 1 (b) corresponds to compensated absorption, $\kappa_{1}=0.1, \kappa_{2} \approx-0.0174, p=100$.

range without violating the causality principle [16]. For this reason, the causality principle does not prevent the compensation of absorption by amplification.

\subsection{Oblique Incidence and Propagating and Evanescent Waves}

The angular dependence of transmission of evanescent waves is especially important in the image transmission problem. The angular dependence of transmission for propagating waves incident on a system $(z=0)$ at an angle of $\theta$ is shown in Fig. 6 (regions to the left of the vertical dashed straight line). It follows from this dependence that the compensation for absorption tends to increase in transmission, but interference oscillations are observed in multilayer systems. Figure 6 also shows the dependences of the transmission modulus on the transverse wavenumber $k_{x}$ for evanescent waves in the case of a system of transparent layers and compensated absorption. We can see that the absorption compensation preserves large transmission coefficients for modes $(\sim 10)$, but these values are finite because, unlike the system of transparent layers, the real values of $k_{x}$ do not correspond to the poles of the transmission function $\tau_{p}\left(k_{x}\right)$ (zeroes of the matrix element $\left.M_{11}\left(k_{x}\right)\right)$. In addition, the transmission coefficient as a function of $k_{x}$ oscillates near the poles of waveguide modes (see Section 4.3 below) corresponding to the complex values of $k_{x}$ located in the upper half-plane. In this case, the closer the compensation conditions to the excitation threshold of waveguide modes, the greater the transmission coefficient for these modes. A finite number of waveguide modes corresponding to the poles $\tau_{p}\left(k_{x}\right)$ of the transmission coefficient is located within a finite interval of $k_{x}$ values. For the specified parameters of a set of numbers, the transmission coefficient approaches zero for $k_{x} d>2.2$, which determines the minimal resolution of images. Under conditions adopted here, $k_{x} d<9.6 k_{0}$, and therefore the value $\delta x \sim \lambda / 9.6$ characterizes the resolu- tion limit, which is independent of the number of layers.

Calculations show that the fulfillment of compensation condition (11) for oblique incidence only for one value $k_{0}$ provides compensation of absorption by amplification in almost the entire first transparency band, and this includes the region of waveguide modes as well.

\subsection{Compensation and Instabilities}

The numerical calculations of the absorption compensation by amplification presented above were obtained by neglecting the possibility of development of instabilities caused by the presence of amplifying layers in the medium. As pointed out above, instabilities of two types should be analyzed: absolute instabilities (lasing at longitudinal laser modes, a discrete set of complex frequencies, $k_{x}=0$ ) and convective instabilities (continuous spectrum of real frequencies, a discrete set of waveguide modes with complex $k_{x}$ for a fixed frequency). The determination of waveguide modes is simplified because the modulus of the imaginary part of the permittivity is small and, hence, perturbation theory over absorption and amplification can be used (see Section 3). The search for laser modes in the general case requires a full consideration of dispersion, i.e., the dependence of the permittivity not only on the real part but also on the imaginary part of the radiation frequency. Note that, as the complex radiation frequency approaches complex frequencies corresponding to the poles of the permittivity of the medium, a complex resonance can be observed [17]. However, the radiation frequency is real on the lasing threshold of interest to us itself. In addition, as mentioned above, the refractive index of media changes weakly within the gain band. These circumstances allow us to search for laser modes assuming that the spectral characteristics of the medium are specified.

Under the conditions considered here, the amplification required to compensate for absorption is always 

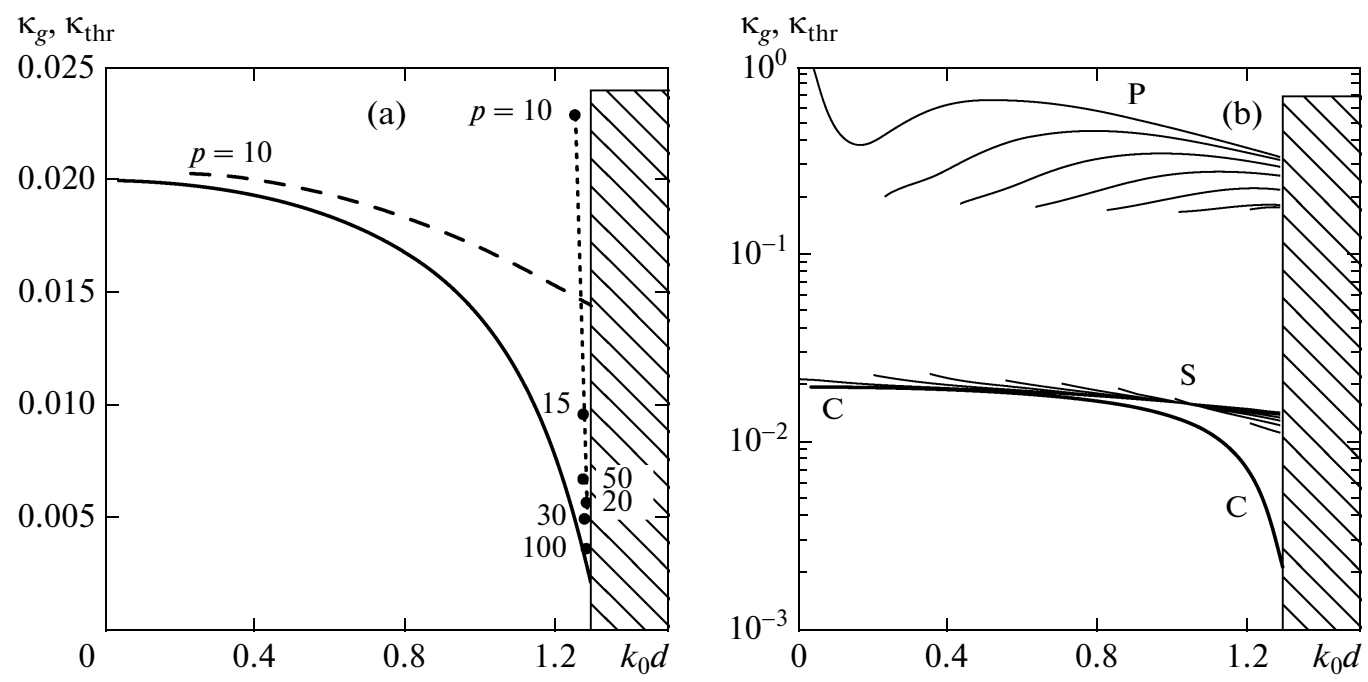

Fig. 7. Frequency dependences $\kappa_{g}$ of the gain in even layers, which is required for the ideal compensation of the specified absorption in odd layers $\kappa_{1} \approx 0.1$ (solid heavy curve), waveguide amplification thresholds (a, dashed curve, $s$-polarization, $p=10$, dependence on $p$ is weak) and lasing (a, dotted curve connecting dark circles at which the number $p$ of periods is indicated). The forbidden band, in which the compensation of absorption by amplification is impossible, is shaded. Amplification required for compensation $(\mathrm{C})$ and waveguide amplification thresholds $(S$, for $s$-polarized waveguide modes and $P$ for $p$-polarized modes) are compared in Fig. $7 \mathrm{~b}$.

lower than the threshold waveguide amplification ( $s$-polarization of perturbations), although the latter approaches it in the limit $p \longrightarrow \infty$. The amplification required for compensation is even smaller than the threshold for $p$-polarized waveguide modes (see Fig. 7). The lasing threshold is noticeably higher for a small number of layers, but it decreases with increasing $p$ and tends asymptotically to a constant value. To obtain the ideal compensation (many periods), this constant level should be higher than the amplification required for compensation.

Figure 7 shows the frequency dependences of amplification required for the ideal compensation and of instability thresholds. The amplification threshold for $s$-polarized waveguide modes is shown in Fig. 7a in simplified form as the envelope over the thresholds of the most unstable modes, which are shown in more detail in Fig. 7b. We can see that $p$-polarized waveguide modes have a higher threshold and are therefore not as important in the problem of absorption compensation by amplification. For low frequencies $\left(k_{0} d \longrightarrow 0\right)$, compensation and amplification thresholds for the $s$-polarized waveguide mode approach each other, although the compensation regime remains stable. More appropriately, in the limit of small phase incursions (the quasi-static approximation) for $s$-polarization, the amplification threshold for $s$-polarized waveguide modes is determined by the condition $\sum_{j} d_{j} \operatorname{Im} \tilde{\varepsilon}_{j}=0$, and a small difference from the compensation threshold is related only to the presence of an additional layer symmetrizing the set of layers. Figure 7 also shows that at higher frequencies (near the edge of the first band), the lasing threshold approaches the compensation threshold in the case of a large number of periods in the system $(p \longrightarrow \infty)$, but the compensation regime still remains stable. Thus, the compensation regime can be achieved in a comparatively broad spectral range covering almost the entire transmission band. To ensure stability of the compensation regime, it is preferable to select the frequency near the forbidden band where the separation from the instability threshold is maximal. To transmit images with superresolution, it is more convenient to compensate absorption for small values of $k_{0} d$ (for example, with the help of thin layers) because in this case the highest transmission coefficient for evanescent waves can be achieved due to the closeness of the amplification threshold for waveguide modes to the compensation condition (see Fig. 6).

\section{CONCLUSIONS}

To ensure propagation of radiation in a system of alternating absorbing and amplifying layers, it is necessary to suppress instabilities: the absolute instability related to lasing and the convective instability appearing in the waveguide amplification of radiation. The propagation and instability regimes are closely interrelated; in particular, the waveguide propagation regimes are responsible for the deep modulation of the transmission coefficient for evanescent waves. Generally speaking, the band transmission structure (alternating allowed and forbidden bands) is broken when absorption or amplification is introduced, the transmission of the system tending to zero with an increas- 
ing number of layers even in systems with amplification. However, the band structure is reconstructed and the transmission coefficient of the system in allowed bands does not tend to zero with an increasing number of layers if the frequency dependence of the gain in active layers is selected to compensate absorption by amplification. Such compensation in forbidden bands is impossible. Conditions are determined under which the compensation is achieved in the absence of lasing and waveguide amplification. To provide transparency in the first allowed band, it is sufficient to obtain compensation near the high-frequency boundary of this band. The large transmission coefficients for evanescent bands required to obtain superresolution can be achieved in the limit of low frequencies or thin layers $\left(k_{0} d \ll 1\right)$ at which the amplification threshold for waveguide modes approaches amplification required to compensate for losses.

\section{ACKNOWLEDGMENTS}

The work was supported by a grant of the Government of the Russian Federation for State Support of Scientific Research Supervised by Leading Scientists in Russian Higher Education Institutions, by the Russian Foundation for Basic Research (project nos. 0902-00848a and 10-02-01016a), the Ministry of Education and Science (grant RNP no. 2.1.1/9824), and the Australian Research Council including Future Fellowship FT100100160.

\section{REFERENCES}

1. L. Brillouin and M. Parodi, Wave Propagation in Periodic Structures (Dover, New York, 1953; Inostrannaya Literatura, Moscow, 1959).

2. O. Svelto, Principles of Lasers (Springer, New York, 1990; Mir, Moscow, 1990).

3. X. Tr. Tran and N. N. Rozanov, Opt. Spectrosc. 104 (4), 558 (2008).

4. S. A. Ramakrishna and J. B. Pendry, Phys. Rev. B: Condens. Matter 67, 201101(R) (2003).
5. S. Xiao, V. P. Drachev, A. V. Kildishev, X. Ni, U. K. Chettiar, H.-K. Yuan, and V. M. Shalaev, Nature (London) 466, 735 (2010).

6. A. Fang, Th. Koschny, and C. M. Soukoulis, Phys. Rev. B: Condens. Matter 82, 121102(R) (2010).

7. S. Wuestner, A. Pusch, K. L. Tsakmakidis, J. M. Hamm, and O. Hess, Phys. Rev. Lett. 105, 127401 (2010).

8. E. I. Kirby, J. M. Hamm, T. W. Pickering, K. L. Tsakmakidis, and O. Hess, Phys. Rev. B: Condens. Matter 84, 041103(R) (2011).

9. D. J. Bergman and M. I. Stockman, Phys. Rev. Lett. 90, 027402 (2002).

10. L. D. Landau and E. M. Lifshitz, Course of Theoretical Physics, Vol. 8: Electrodynamics of Continuous Media (Nauka, Moscow, 1982; Butterworth-Heinemann, Oxford, 1984).

11. M. Born and E. Wolf, Principles of Optics: Electromagnetic Theory of Propagation, Interference, and Diffraction of Light (Pergamon, Oxford, 1964; Nauka, Moscow, 1970).

12. L. D. Landau and E. M. Lifshitz, Course of Theoretical Physics, Vol. 6: Fluid Mechanics (Nauka, Moscow, 1986; Butterworth-Heinemann, Oxford, 1987).

13. N. N. Rosanov, ${ }^{1}$ Opt. Spectrosc. 106 (3), 430 (2009).

14. L. A. Mel'nikov and O. N. Kozina, Opt. Spectrosc. 94 (3), 411 (2003).

15. L. D. Landau and E. M. Lifshitz, Course of Theoretical Physics, Vol. 3: Quantum Mechanics: Non-Relativistic Theory (Nauka, Moscow, 1974; Butterworth-Heinemann, Oxford, 1981).

16. B. Nistad and J. Skaar, Phys. Rev. E: Stat., Nonlinear, Soft Matter Phys. 78, 036603 (2008).

17. N. N. Rosanov, ${ }^{1}$ JETP 111 (4), 534 (2010).

Translated by M. Sapozhnikov

\footnotetext{
${ }^{1}$ This is another transliteration of the same author's name "Rozanov."
} 